\title{
Review
}

\section{Toxic effect onset and evaluations of medicinal drugs - horizon for Darwinian toxicological thought -}

\author{
Ikuo Horii1,2 \\ ${ }^{1}$ Showa University (Department of Biochemical Toxicology, School of Pharmaceutical Sciences), \\ 1-5-8 Hatanodai, Shinagawa-ku, Tokyo 142-8555, Japan \\ 2Pfizer (Global Research \& Development, Drug Safety Research \& Development), \\ Shinjuku Bunka Quint Bldg., 3-22-7, Yoyogi, Shibuya-ku, Tokyo 151-8589, Japan
}

(Received May 19, 2010)

\begin{abstract}
The theory of Darwinian Medicine linked to an extension of Darwin's evolutionary theory is based on the approach from the aspect of "why we become ill?". This theory enables us to understand the relationship between humans and diseases by thinking from evolutional perspective, shows an important help for preventive medicine, and is meaningful to consider the future human healthcare. Toxicology has been defined as a research of adverse effect of xenobiotic substances backed up by diverse-sciences. Toxic effects are basically responses to xenobiotic substances, and expressed as triggering or additional accelerating adverse effects toward abnormal condition. Toxic effects, biological adverse responses, are interpreted as protective responses of living body, and the adverse effects caused by drugs are also considered to be protective responses. This logic can be translated as "Darwinian Toxicology" corresponding to "Darwinian Medicine", replying to "why we get into toxic condition by xenobiotics exposure". This paper refers to the meaning of toxic effects based on mechanisms underlying and comprehensive drug safety evaluation from Darwinian Medicine perspectives.
\end{abstract}

Key words: Darwinian toxicology, Mechanistic toxicology, Drug safety evaluation

\section{INTRODUCTION}

The theory of Darwinian (or evolutionary) Medicine has been increasingly advocated since the 1990s. Whereas the approach pursued in traditional medicine has been to answer the questions of what causes diseases and how we become ill, this theory is based on an approach from the aspect of why we become ill. The proposition that most diseases are caused by the relationship between genetic and environmental factors is linked to an extension of Darwin's theory of evolution. In addition, the theory of Darwinian Medicine readily enables us to develop a logical framework to understand the relationship between humans and diseases by thinking about diseases from an evolutionary perspective, showing the potential to be of great assistance in the field of preventive medicine and relevant to the future of human healthcare.

Toxicology has been defined as research on the adverse effects of xenobiotic substances, and is supported by diverse other fields of science (multi-disciplinary science); namely we are obliged to make an approach for toxicity evaluation from a variety of scientific standpoints. Recently, in the field of toxicology, scientists have been researching how resistance to toxicants can be enhanced by new science and technology in the field of molecular biology. Toxic effects are basically responses to xenobiotic substances (drugs), expressed as a triggering or acceleration of a biological processes leading from a normal to an abnormal condition. The similar logic is reflected in the relationship between Darwinian Toxicology and Darwinian Medicine. A toxic effect, an adverse biological response, is interpreted as a protective response of the living body, so an adverse effect caused by a drug could also be considered to be a protective response in the broad sense of the term. This paper discusses the positioning of mechanisms of expression of toxic effects, interpretation and evaluation of toxicity study results, and comprehensive drug safety evaluation from such a perspective.

Correspondence: Ikuo Horii (E-mail: ikuohorii@hotmail.com, ikuo.horii@pfizer.com) 


\section{Background to the Proposition of Darwinian Medicine}

For a variety of diseases, symptoms have been revealed to be protective reactions of the living body, and genes that had been thought to cause diseases have come to be seen as beneficial to human survival. This kind of thinking, which underpins the theory of Darwinian Medicine, was pioneered by researchers in the medical field such as Paul Eward and Randolph Nesse, and an evolutionary biologist, George Williams in the 1990s (Nesse and Williams, 1995). In traditional medicine, scientists have researched what causes diseases or how we get ill by elucidating originated genes and their expression modes. However, this approach has shortcomings when considering diseases from the perspective of why we get ill (Lappe, 1994). In Darwin's theory of evolution (Darwin, 1859), all living organisms are thought to be results of the evolutionary process of natural selection, in which it is of great importance whether the direction of mutation is toward an allelic gene that confers resistance to a particular disease. Genes are involved in morphogenesis and the functional expression of each organ within the biological body, and the elimination of unfavorable characters (genes) over generations (Zimmer, 2007). Since natural selection is in the category of biological adaptation, it is not so difficult to understand biological events from a medical standpoint in the context of evolution. In this way, medical researchers can find it useful to understand diseases and to explain infections, toxication, injury, genetic diseases, allergy, cancer, mental disorders, childbirth, adult diseases, and aging based on research into evolutionary medicine, and there is also a benefit for preventive medicine. It is important to see disease components from an evolutionary perspective in order to understand the relationship between humans and disease, and it is also meaningful to think about the future relationship between humans and disease. As Imura (2006, 2008) has said, "Evolutionary medicine is a learning methodology in which an understanding of the mechanism of disease is gained from an evolutionary perspective rather than through its provisions." At any rate, the proposition that most diseases are caused by a relationship between hereditary factors and environmental factors is linked to an extension of the theory of evolution that Darwin propounded.

\section{Steps toward Darwinian Toxicological Thought}

Toxicology has been defined as research on the adverse effects of xenobiotic substances. Disciplinary boundaries of toxicology are related to many diverse fields of supporting research, and a combination of approaches from various standpoints, such as pathology, pharmacology, biochemistry, and physiology is required. In a recent toxicology, scientists have researched on related corresponding changes in toxicant by using new science and technology on molecular biological sciences. Namely, an approach in which molecular toxicology is supplemented by many other scientific disciplines has been advocated, and is widely accepted at the present time. It may be no exaggeration to say that toxic effect expression of drugs exists in this thinking category. The mechanisms of toxicity expression are generally understood to function in the following four steps (Klaassen, 2008):

1) Exposure to a toxicant (processes of delivery to the target-site: absorption, distribution, excretion, reabsorption, detoxification, and toxication),

2) Reaction of toxicant with target molecules (reaction pattern: non-covalent binding, covalent binding, dehydrogenation, electron transfer, and enzymatic reaction; response of target molecules: functional disorder, destruction, and immune response)

3) Cellular dysfunction and resultant toxicities (cellular dysfunction: dysregulation of gene expression and ongoing cellular activity; impairment of cellular maintenance: cell maintenance disorder and function maintenance disorder internal/external to the cell)

4) Recovery and recovery disorder (molecular recovery: protein, fat, and DNA repair; adaptation: increasing/decreasing repair; cell/tissue repair: degeneration, necrosis, fibrosis, apoptosis, proliferation, and carcinogenesis).

Taking these toxic effects into consideration from the aspect of organoid, cell, tissue or organ, above toxic process of series in toxic effects means defensive reactions against xenobiotic substances in living body. Such a defensive reaction is a normal phenomenon for toxicity proceeding from homeostatic function maintenance to disorder expression (functional and organic disorders), and ultimately to death when viewed from each perspective. Thinking from this point of view, it can be said that toxic effects caused by a xenobiotic substance initially trigger or further accelerate the process from a normal toward an abnormal condition. This logic can also be seen as underpinning the relationship between Darwinian Toxicology and Darwinian Medicine. The adverse biological reaction, the toxic effect, is a defensive reaction of the body, and side effects induced by drugs are also defensive reactions in the broad sense of the term. Moreover, it may be no exaggeration to say that these genes (gene expression thought to trigger toxic effect expression) are working 
Darwinian toxicology thought in safety evaluation of drug

to maintain the living body or seeking some advantage for its continuance (Bickham and Smolen, 1994). With employing risk assessment or risk management to evaluate the toxic effects of a drug toxic from the perspective of Darwinian Toxicology, it is possible to manage drug safety evaluation, and to reach the correct judgment on whether the relevant toxic effects are critical or manageable.

Traditional toxicology focuses on a pursuit of what causes toxic effect, and how we can fall into a toxic condition, and recently scientists have sought explanations for the causal mechanisms from new areas of scientific research such as molecular toxicology. However, these approaches by themselves are not sufficient to explain why we can fall into a toxic condition after exposure to a xenobiotic. The approach of Darwinian Toxicology is to research how drugs can work as a trigger for toxic effects, and how they influence the induction of toxic effects. This approach could prove to be a fruitful way to understand complicated toxic effects from a stable viewpoint for appropriate risk assessment and risk management (Fig. 1).

\section{Mechanisms Underlying Toxic Effect}

Prior to the emergence of the theory of Darwinian Toxicology, it was essential to identify/clarify the toxic effect and its mechanism from diverse scientific perspec- tives for drug safety evaluation (Boelsterli, 2007). The mechanism for the appearance of toxicity is considered from various perspectives in the sections outlined below.

\section{Mechanism for the appearance of toxicity from a general toxicological perspective}

The toxic effect of a drug and its level can vary greatly depending on exposure (amount of exposure and period for which it is sustained) at the target site. The reaction between the target molecules and the toxic effect-inducing substance at the target site are expressed in patterns (noncovalent binding, covalent binding, dehydrogenation, electron transfer, enzymatic reaction, etc.). After exposure to a compound, toxic expression begins as it interacts with target molecules, and this is followed by biological change toward the appearance of toxicities such as functional disorder, destruction, regeneration, and disruption of cells. Cells in the living body are constantly participating in function adjustment. If toxic effects extend to targets involved in cellular regulation including the signaling system, toxicities corresponding to impaired expression of genes (improper expression: cell proliferation to tumor formation, apoptosis to cell involution and teratogenesis, protein synthesis to peroxisome proliferation, etc.) or cell function maintenance abnormality (improper neuromuscular activity: tremor, paralysis, insult, cardiac arrhythmia, and paresthesia) may appear. In the case of a toxic effect on cell maintenance, intra-cellular (cell

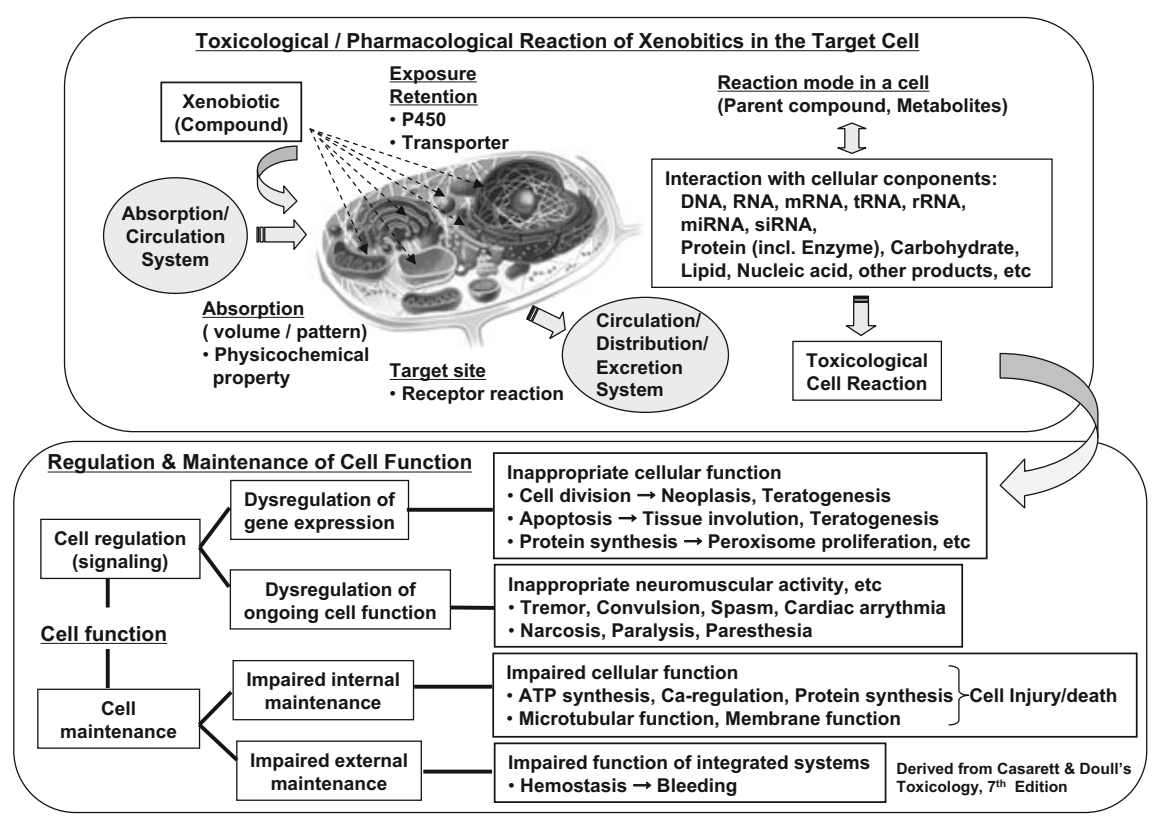

Fig. 1. Toxicological/Pharmacological Action-Process in a Cell. 
disorder and necrosis: disorders of ATP synthesis, calcium ion control, protein synthesis, micro-tubular function, and cell membrane function), or extra-cellular functional disorder (total system functional disorder: hemostasis and bleeding) is expressed.

\section{Mechanism for the appearance of toxicity from diverse scientific perspectives}

From the cytological perspective of efficacy and toxic effect in target cells (Fig. 1):

Compounds are considered as xenobiotics for the living body, and toxic effects may vary greatly depending on to how much and for how long the relevant target cells are exposed. The pattern of exposure to the compound for the relevant target cells is determined from the ways of uptake (trans-membrane absorption: transporters, involvement of circulatory system: distribution by circulating blood), subsistence (involvement of drug-metabolizing enzymes), and excretion (trans-membrane excretion: transporters; involvement of the excretory system: excretion in the blood, bile, and/or urine). Unchanged drugs or various metabolites have an action on the target receptors in cells, and a toxic effect is induced by this interaction; excessive responses or amounts of functional substances in cells (DNA, RNA, mRNA, tRNA, miRNA, siRNA, protein, lipids, and carbohydrates) can appear. In addition, such toxic effects are often manifested as organic disorder in cells.

From the pathological/physiological perspective of histopathlogical reaction (Fig. 2):

Following exposure to primary stimuli (xenobiotics), foreign substance recognition, physiological function change, endocrinological change, and organic damage are systemically seen as histopathological and physiological reactions against foreign substances in the living body. After the initial reaction to foreign substance recognition acts as a trigger, leukocyte infiltration/migration, phagocytosis, and production of antibodies against the foreign substance are induced, followed by tissue/organ changes such as vascular and intimal inflammatory changes, encapsulation formation, and cell degeneration. Moreover, vascular contraction/expansion, hyperemia, and hyperplastic changes occurring as functional/endocrinological reactions become advanced or organic changes. Further consequences are the emergence of degeneration, necrosis, and abnormal cell differentiation, and sometimes carcinogenesis and teratogenesis. The recovery process of changing back to a normal condition progresses in parallel with the above-mentioned toxicological changes as reinforcement of degeneration, necrosis, and phagocytosis in the abnormal region.
From the pharmacological/toxicological/pharmacokinetical perspective of toxicity onset (Fig. 3):

Various target cells play a role in gene regulation through the process (absorption, distribution, metabolism, and excretion) of elimination of xenobiotics. The cellular incorporation of a xenobiotic causes reactions with target molecules at the receptor level through gene regulation, and is expressed as a pharmacological effect. If the action is excessive and it falls into the category of a toxic reaction as a cell functional disorder or cell disruption, it is expressed as a toxic effect. In parallel with induction of a toxic effect by a cell disorder, body function disorders are seen, followed by recovery reactions to eliminate the disorder. By going through this series of reactions looking at the pharmacokinetics (PK) of the compound as absorption, distribution, metabolism, and excretion, an understanding of the conditions of exposure (concentration and time to saturation) at the target site is gained, and efficacious and toxic effects are defined pharmacologically and toxicologically as functional disorder, tolerance, disruption, and recovery, which are all part of the body's defenses.

From the biochemical perspective of body reaction based on molecular biological research:

A biochemical approach from the standpoint of molecular biology is necessary to elucidate the mechanisms for body defense, tolerance, disruption, and recovery after exposure to a xenobiotic. Considering the biological meaning of the toxic effect (from the standpoint of molecular toxicology) based on the biochemical reactions in target cells, the mechanisms of toxic effect expression can be classified broadly as follows:

- Transfer in the cells and preferential accumulation of toxic effect-inducing substance

- Biological activation of metabolization

- Oxidative stress: cellular disorder, signal transduction, and gene regulation

- Disorder and deterioration of cellular calcium homeostasis

- Necrotic and apoptotic cell death

- Functional disorder in cell growth and tissue repair

- Covalent linkage between active metabolite and cells, high molecular mass

- Immunological function disorder

- Disorder related to cytokines

- Specific inactivation of enzymes and other proteins

- Disorder related to nuclear receptors

- Interaction with transporters

- Cell energy production disorder

- Individual gene expression disorder

Detailed information is laid out in the references con- 
Darwinian toxicology thought in safety evaluation of drug

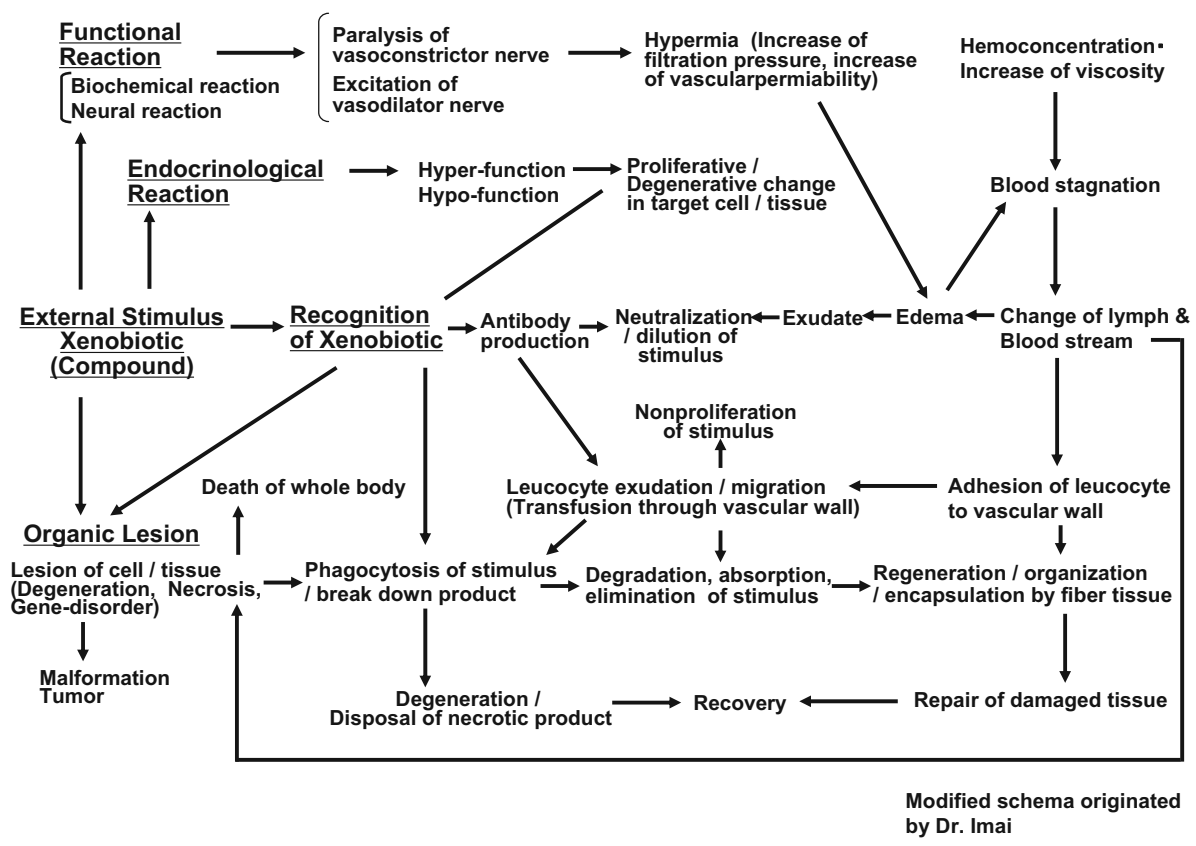

Fig. 2. Histopathological Changes in Toxicological Reaction: From the aspect of toxicological pathology \& physiology.

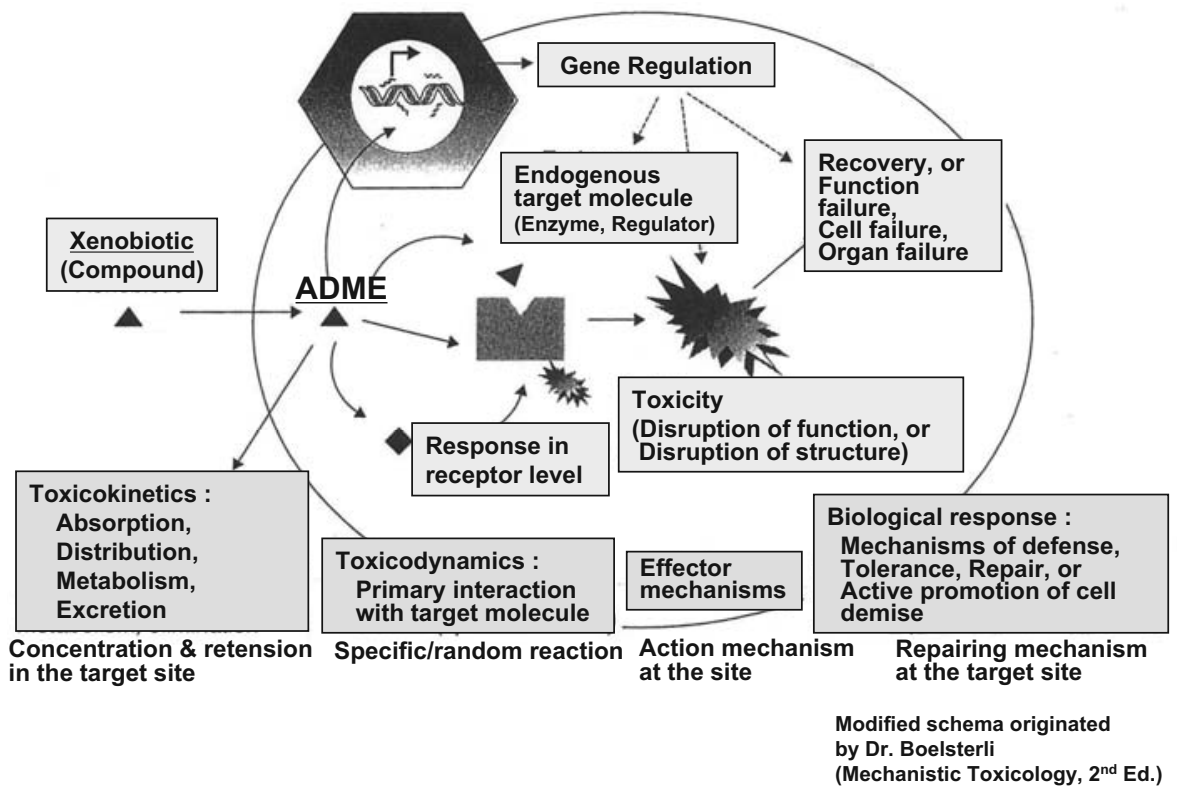

Fig. 3. Onset of toxicological effects: From the aspect of pharmacology, toxicology, pharmaco/toxicokinetic aspects.

cerned (Nelson and Pearson, 1990; Bursch et al., 1992; Boelsterli, 1993; Buja et al., 1993; Baumann and Gauldie, 1994; Raghow, 1994; Hardman et al., 1995; Cohen et al., 1997; Wyllie, 1997; Darzynkiewicz et al., 1998; Kroemer et al., 1998; Toivala and Eriksson, 1999; Daniel, 2000; Wallace and Starkov, 2000; Blatt, 2001; Williams, 2001; Boatright and Salvesen, 2003; Roth et al., 2003; McGowan and Russell, 2004; Meijer and Codogno, 2004; Moggs 


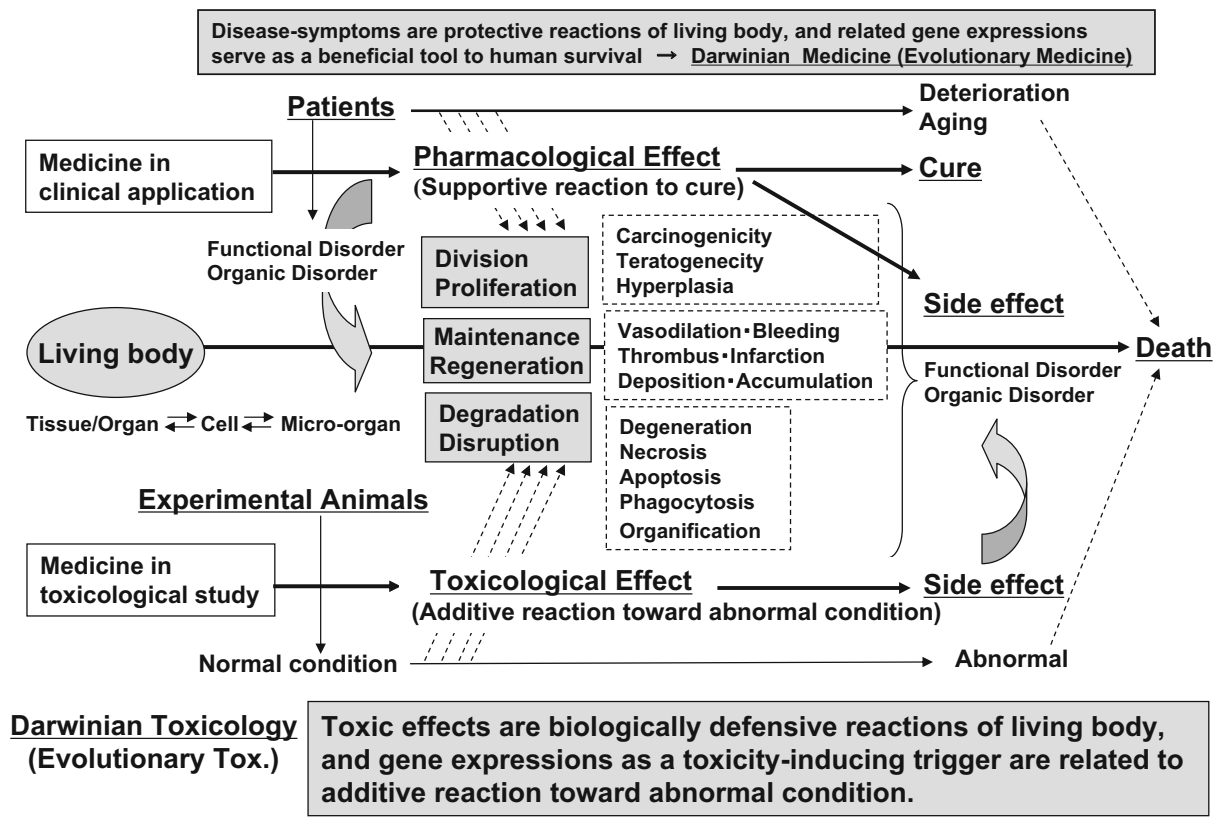

Fig. 4. Darwinian Toxicological Thought in Medicine Toxicity.

and Orphanides, 2004; Orrenius, 2004; Cribb et al., 2005; Mehendale, 2005; Rhee et al., 2005).

\section{Application of Darwinian Toxicology to Drug Safety Evaluation}

Overall view of Darwinian Toxicology thought is shown in Fig. 4. Darwinian Toxicology focuses attention on understanding and evaluating toxicological study results for the relevant compound. As a result of considering the toxic effect expression mechanisms above from diverse scientific perspectives, events that a living body recognizes compounds (drugs) as foreign substances lead to functional and organic changes themselves. The outcome is an additional, accelerated reaction in a normal biologically living body (organoid cells, cells, organs, and tissues), as a toxic effect against the process of maintenance, regeneration, division, proliferation, degradation, and disruption. The biological reaction, the toxic effect, is expressed as a defensive reaction of the body, and gene expression is thought to trigger the induction of the toxic effect for the purpose of body maintenance or survival. Reactions related to homeostatic maintenance and regeneration are shown as changes such as vasodilatation, bleeding, thrombus, infarction, deposition, and accumulation, changes related to decomposition and disruption are expressed as degeneration, necrosis, apopto- sis, phagocytosis, and organification, and changes related to division and proliferation are shown as carcinogenesis (tumorigenic transformation), teratogenesis (developmental anomaly), and hyperplasia (accelerated proliferation). It is important to clarify the generation of toxic change and the action mechanism with an approach based on Darwinian Toxicology looking at drug exposure.

\section{Interpretation and evaluations of toxicity study results}

The aim of a toxicity study for drug safety evaluation is to clarify what toxic effect is induced and the corresponding exposure level that it is induced at, and to determine the highest level of exposure at which there is no toxic effect (no-observed-adverse-effect level). It is necessary to make a judgment on the relationship between exposure and toxic effect, on which changes are actually toxic effects, and on whether the toxic effect is critical. Moreover, an overall safety evaluation of the toxicity needs to consider its grade, action mechanism, reversibility, therapeutic index (TI), and clinical relevance.

Considering from the idea of Darwinian toxicological perspective, "pharmacological effect as efficacy" and "toxicological effect as adverse effect" exist on consequence line. "How induced toxic effect is explained from Darwinian toxicological perspective" is meaningful for safety evaluation of the drug. 
Darwinian toxicology thought in safety evaluation of drug

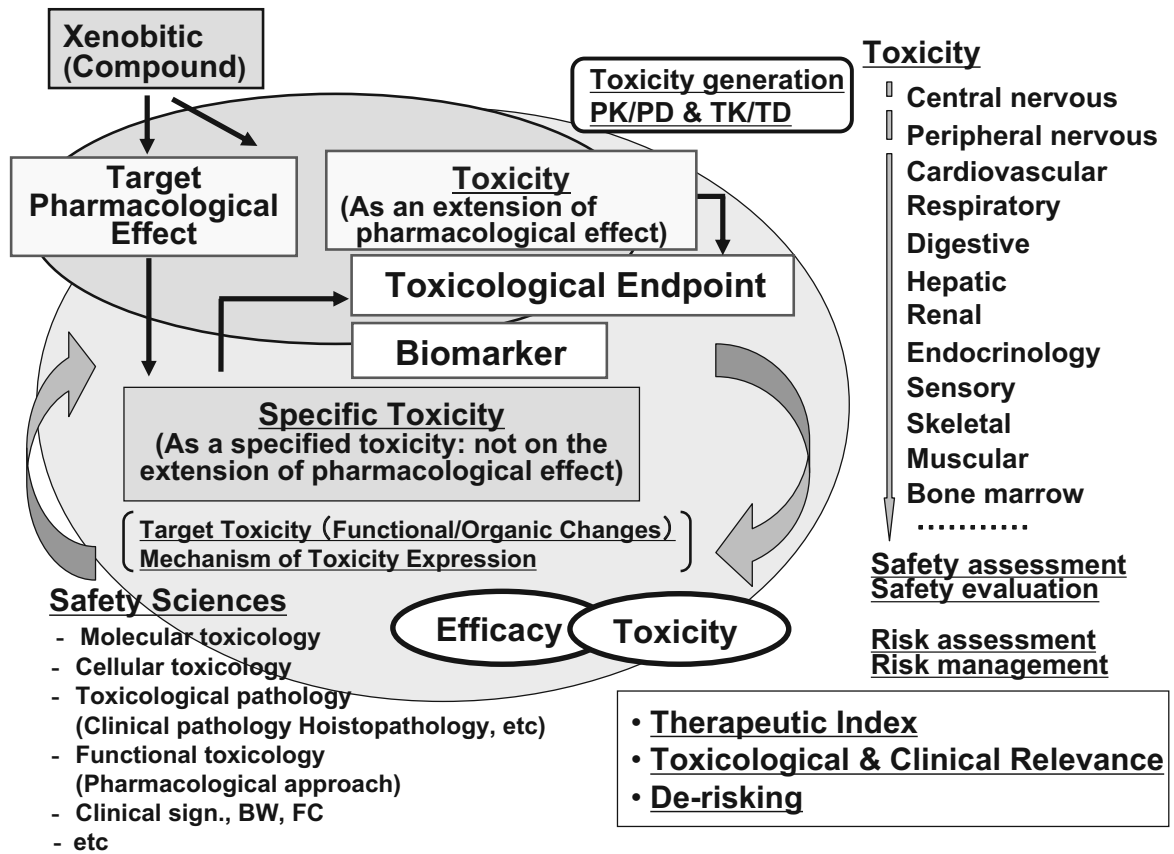

Fig. 5. Safety Assessment \& Evaluation in Safety Studies.

\section{Identification of toxic effect}

A safety evaluation in a toxicity study starts by trying to identify the toxic effect. The first step is to ascertain what toxic effect there was by scientifically reviewing the results (findings and changes) of the toxicity study. Initially, changes that statistically significant differences are seen, and that deviate from the limit of background data and spontaneously arising findings as physiological variation should be flagged as "findings and changes" that thought to be possible to have relationship with drug administration. Among them, changes that have statistically significant difference with dose-correlation and changes greatly deviated from physiological variation or background data should be evaluated as "toxicologically significant changes". When seeing specification of toxic effect from Darwinian toxicological perspective, "specified change" means clear additional and accelerating reactions toward abnormal condition as a foreign substance recognition and its responding action.

Generally, the biological reaction induced by a foreign substance is classed as an efficacious or an adverse effect. When viewed from the perspective of the target of the biological reaction, toxic effects are divided into those that are an extension of efficacy, and those that are not an extension of efficacy. When the target corresponds to the pharmacological target, the induced change can be understood as an extension of efficacy, and when the toxic effect is not induced on the pharmacological target, it is generally understood not to be an extension of efficacy. It is often difficult to draw a line between these two classifications because the targets can appear to come into both categories (Fig. 5). To fully resolve this dilemma, a diverse scientific approach utilizing the latest developments in science and technology will be needed. It is meaningful to clarify the mechanism of expression for the toxic effect expression and how the relevant drug adds and accelerates the process to induction of the toxic effect based on the thinking embraced by Darwinian Toxicology. This basic idea will lead to and develop systems biology and systems toxicology (Hirabayashi and Inoue, 2008).

In the case that the potential occurrence of critical change in humans such as direct gene disorder (genotoxicity, carcinogenicity, and teratogenesis), living function disorder (sensory toxicity), or an irreversible toxic effect (severe organic disorder) is observed as a critical toxic effect in a toxicity study, it is always difficult for judging whether continues the ongoing clinical development as long as the mechanism is being clarified about human relevance. When the toxic effects are not critical, it is permissible to proceed to develop in consideration of the appropriate exposure conditions such that the induction of toxic effects is in a category capable of being controlled 


\section{Toxico-DMPK Panomics Approach}

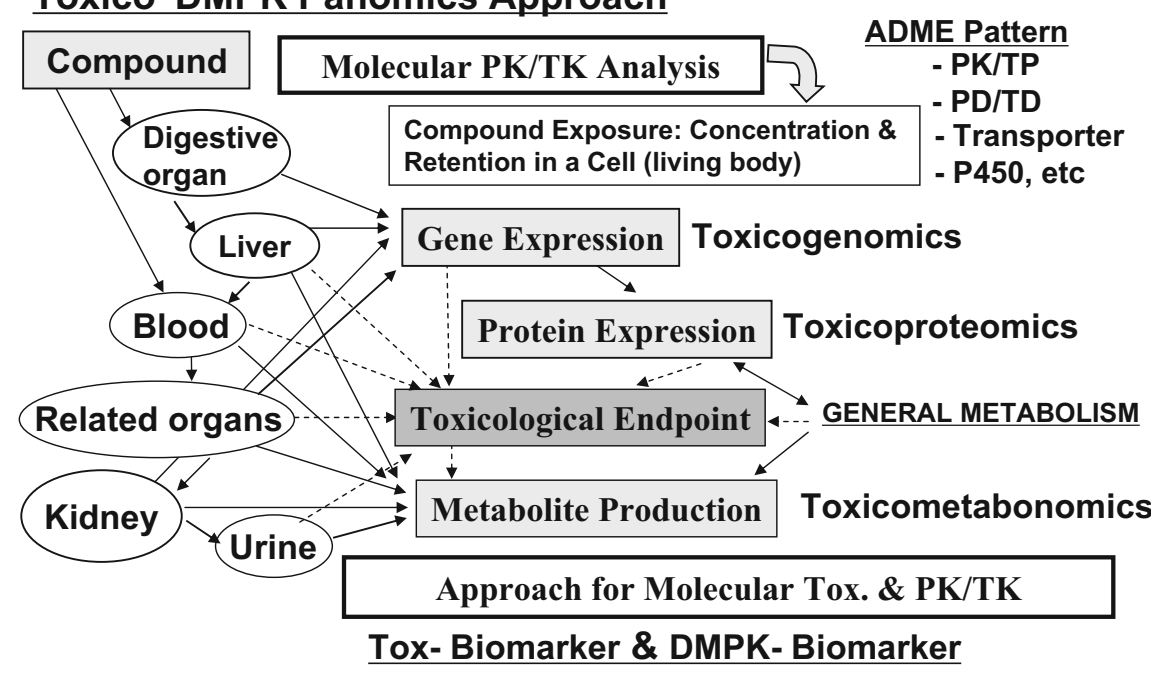

Fig. 6. Molecular Toxicology and Molecular DMPK.

by gene expression. Pharmacokinetics (PK; absorption, distribution, metabolism, and excretion) profiles from toxicokinetics (TK) data assist in making a reasonable evaluation of safety in humans. It is essential to evaluate the relationship between toxicity and drug exposure from the perspective of systemic exposure in any consideration of safety (Sugiyama et al., 1996; Horii, 1999). A typical drug safety study (toxicology study) is conducted based on traditional administrative doses; however, we can get a more rational safety assessment by considering blood level (AUC and Cmax) indices. In some specific cases such as saturation of absorption, metabolism, or excretion, drug metabolism induction, accumulation, or aging effect, TK parameters are not always linked to administrative doses. Basically, once administered, drugs are absorbed at the relevant site, distributed to tissue through blood circulation, and they reach the target site and cause a pharmacological or toxic effect. The strength of the efficacy/ toxicity is thought to be determined by the drug exposure level, and also partially by the sensitivity of the expression site. In addition, it is necessary to quantify the volume excreted during distribution pharmacokinetically and to consider the active metabolite (Lin et al., 2003; Ekins et al., 2005), (Fig. 6). Taking exposure into consideration based on PK/TK, the theory of Darwinian Toxicology can be applied to the toxic effects to make a risk assessment and a consideration of risk management for any additional and accelerating responsive reaction in the living body (homeostatic maintenance reaction, regeneration, division, proliferation, and degradation).

\section{Overall safety evaluation: safety evaluation from the clinical viewpoint}

The determination of the mechanism of toxicity expression, the grade of expression, and the reversibility of toxicity are important for an evaluation of safety in humans. The corresponding adverse effects predicted from an extrapolation to humans should be considered. It is essential to explain the mechanism of toxic expression in order to demonstrate whether or not a toxic effect is specific to an animal species, and what is its relevance to humans.

The level of expression can generally be quantified as a toxic or non-toxic level, but the judgment on this level could differ depending on the type of toxicity expressed. Besides safety evaluation, consideration is often given to the whether the drug is novel and represents an innovation. When a drug has a revolutionary pharmacological effect, some degree of toxic effect can be accepted for human application. In such an evalaution, the recovery after a cessation of administration (whether it recovers quickly, whether it takes long time to recover, or it does not recover at all) can be an important point.

Another important index when conducting a clinical trial is the safety margin, which is actually evaluated as the TI. The TI in a clinical trial is usually derived by dividing a non-toxic exposure level for target toxicity by the estimated effective blood concentration in humans. The TI is set for the dose to be applied corresponding to each drug indication and type of toxic effect. Even in a case of the same toxic effect, TI can vary greatly depending on the indication. Considering that a toxic effect seen 
Darwinian toxicology thought in safety evaluation of drug

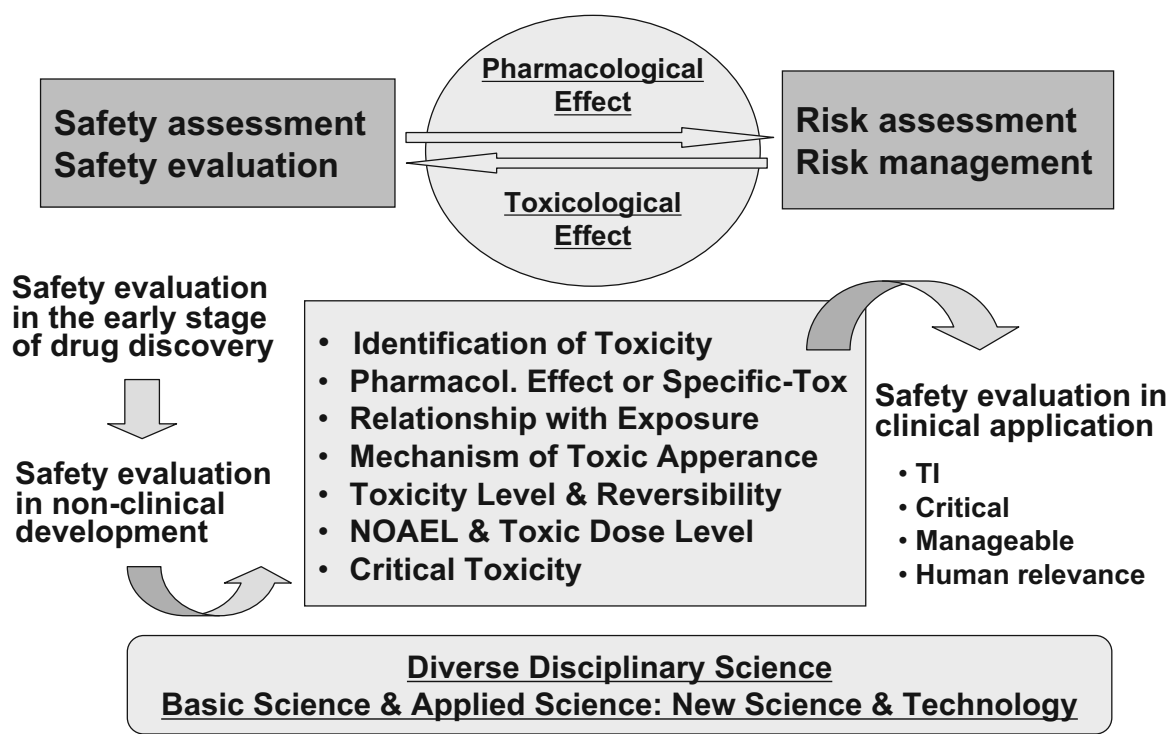

Fig. 7. Safety Assessment/Evaluation for Risk Assessment/Management.

in animals can appear in human as an adverse effect, the setting of appropriate biomarkers to predict the expression of the adverse effect, and procedures for emergency treatment of the adverse effect are critical requirements for safety control from a clinical perspective. In short, if a minimum TI is secured, appropriate biomarkers are set, and an emergency treatment procedure is established, it can be possible to conduct a clinical trial smoothly without further restrictions attached (Fig.7).

The establishment of a comprehensive system for the evaluation of parameters based on toxic effect expression mechanism, body exposure, therapeutic index, and biomarker detection is expected in the future safety evaluation. By combining with an interpretation of toxicity data in nonclinical study from the perspective of Darwinian Toxicology and an interpretation of clinical trial data from the perspective of Darwinian Medicine, approach for systems biology/toxicology is highly desirable to open the door for ultimate level of safety risk assessment and management.

\section{CONCLUSION}

The toxic effect of a drug is expressed as a defensive reaction by the body against a foreign substance. The toxic effect caused by a foreign substance can be said to be a trigger to an abnormal condition, or additional or accelerating action leading to such an abnormal condition in contrast with the course of normal function maintenance to avoid abnormal conditions. This can be seen as the logical framework that links Darwinian Toxicology as a response to Darwinian Medicine. The biological reaction, the toxic effect, is expressed as a defensive reaction in the body, thus the induced adverse effect caused by the drug can also be seen as a defensive reaction in the broad sense of the term. It is thought that, with risk assessment and risk management, a judgment can be made as to whether the toxic effect of the relevant drug is critical or manageable, and as to how to avoid risks of drug toxicity in an evaluation from the perspective of Darwinian Toxicology.

In recent years, scientists have strived to clarify toxic effect expression mechanisms with the introduction of new fields of research such as molecular toxicology. It is important to approach an evaluation of a compound from the angle of why exposure to it causes toxic effect expression (Waring and Ulrich, 2000; Pognan, 2004; Waters and Fostel, 2004; Orr, 2006; Waters and Yauk, 2007). Darwinian Toxicology helps us to easily understand how drugs work as triggers of toxic effects and how toxic effects are induced, in a drug safety evaluation. It offers the prospect of a valuable method to consider the reaction to toxic effects for which appropriate risk assessment and risk management would otherwise prove complicated. 


\section{ACKNOWLEDGMENTS}

This article is based on a lecture entitled "Toxic Effect and Evaluation of Medicinal Drugs: Horizon for Darwinian Toxicological Thought" which I was invited to deliver at the $36^{\text {th }}$ Annual Meeting of the Japanese Society of Toxicology (2009). I would like to express my deep thanks and appreciation to Prof. Shuji Tsuda (Iwate University) who gave me the opportunity to present these ideas at that meeting, Dr. Tohru Inoue (National Institute of Health Sciences) with whom I discussed these theories and from whom I have learned much about them, and Prof. Yasushi Yamazoe (Tohoku University) who kindly spared the time to review this article.

\section{REFERENCES}

Baumann, H. and Gauldie, J. (1994): The acute phase response. Immunol. Today, 15, 74-80.

Bickham, J.W. and Smolen, M.J. (1994): Somatic and heritable effects of environmental genotoxins and the emergence of evolutionary toxicology. Environ. Health Perspect., 102, 25-28.

Blatt, N.B. and Glick, G.D. (2001): Signaling pathways and effector mechanisms pre-programmed cell death. Bioorg. Med. Chem., 9, 1371-1384

Boatright, K.M. and Salvesen, G.S. (2003): Mechanisms of caspase activation. Curr. Opin. Cell Biol., 15, 725-731.

Boelsterli, U.A. (1993): Specific targets of covalent drug-protein interactions in hepatocytes and their toxicological significance in drug-induced liver injury. Drug Metab. Rev., 25, 395-451.

Boelsterli, U.A. (2007): Mechanistic Toxicology - The Molecular Basis of How Chemicals Disrupt Biological Targets. 2 ${ }^{\text {nd }}$ Edition, CRC Press Taylor \& Francis Group.

Buja, L.M., Eigenbrodt, M.L. and Eigenbrodt, E.H. (1993): Apoptosis and necrosis. Basic types and mechanisms of cell death. Arch. Pathol. Lab. Med., 117, 1208-1214.

Bursch, W., Oberhammer, F. and Schulte-Hermann, R. (1992): Cell death by apoptosis and its protective role against disease. Trends Pharmacol. Sci., 13, 245-251.

Cohen, S.D., Pumford, N.R., Khairallah, E.A. Boekelheided, K., Pohle, L.R., Amouzadehe, H.R. and Hinsonb, J.A. (1997): Contemporary issues in toxicology: Selective protein covalent binding and target organ toxicity. Toxicol. Appl. Pharmacol., 143, 112

Cribb, A.E., Peyrou, M., Muruganandan, S. and Schneider, L. (2005): The endoplasmic reticulum in xenobiotic toxicity. Drug Metab. Rev., 37, 405-442.

Daniel, P.T. (2000): Dissecting the pathways to death. Leukemia, 14, 2035-2044

Darwin, C. (1859): The origin of species by means of natural selection or the preservation of favoured races in the struggle for life. Penguin Books.

Darzynkiewicz, Z., Bedner, E., Traganos, F. and Murakami, T. (1998): Critical aspects in the analysis of apoptosis and necrosis. Hum. Cell, 11, 3-12.

Ekins, S., Nikolsky, Y. and Nikolskaya, T. (2005): Techniques: Application of systems biology to absorption, distribution, metabolism, excretion and toxicity. Trends in Pharmacol. Sci.,
26, 202-209.

Hardman, J.D., Gilman, A.G. and Limbird, L.L. (1995): Goodman \& Gilman's The Pharmacological Basis of Therapeutics, $9^{\text {th }}$ ed. McGraw-Hill, New York.

Hirabayashi, Y. and Inoue, T. (2008): Principle of data mining in toxicogenomics-Toxicogenomics a powerful tool for toxicity assessment. (Sahu, S.C., ed.), by Sahu, S.C., 57-84.

Horii, I. (1998): Advantages of toxicokinetics in new drug development. Toxicol. Lett., 657-664.

Imura, H. (2006): Why human gets sick - From the aspect of evolutionary medicine. (Original source is Japanese), Iwananishoten, Japan.

Imura, H. (2008): Obesity, Diabetes, Life-span, understandable by evolutionary medicine. (Original source is Japanese), Iwanamishoten, Japan.

Klaassen, C.D. (2008): Casarett \& Doull's Toxicology The basic Science of Poisons. $7^{\text {th }}$ Edition, McGraw-Hill, New York.

Kroemer, G., Dallaporta, B. and Resche-Rigon, M. (1998): The mitochondrial death/life regulator in apoptosis and necrosis. Annu. Rev. Physiol., 60, 619-642.

Lappe, M. (1994): Evolutionary Medicine: Rethinking the Origin of Disease. Sierra Club Books.

Lin, J., Sahakian, D.C., de Morais, S.M., Xu, J.J., Polzer, R.J. and Winter, S.M. (2003): The role of absorption, distribution, metabolism, excretion and toxicity in drug discovery. Curr. Top. Med. Chem., 3, 1125-1154.

McGowan, C.H. and Russell, P. (2004): The DNA damage response: sensing and signaling. Curr. Opin. Cell Biol., 16, 629-633.

Mehendale, H.M. (2005): Tissue repair: An important determinant of final outcome of toxicant-induced injury. Toxicol. Pathol., 33, 41-51.

Meijer, A.J. and Codogno, P. (2004): Regulation and role of autophagy in mammalian cells. Int. J. Biochem. Cell Biol., 36, 2445-2462.

Moggs, J.G. and Orphanides, G. (2004): The role of chromatin in molecular mechanisms of toxicity. Toxicol. Sci., 80, 218-224.

Nelson, S.D. and Pearson, P.G. (1990): Covalent and noncovalent interactions in acute lethal cell injury caused by chemicals. Annu. Rev. Pharmacol. Toxicol., 30, 169-195.

Nesse, R.M. and Williams, G.C. (1995): WHY WE GET SICK The New Science of Darwinian Medicine. Vintage Books, a division of Random House, Inc., New York.

Orr, M. (2006): Toxicogenomics and cross-species biomarker discovery: Applications in drug discovery and safety assessment. Toxicol. Mech. Methods, 16, 79-87.

Orrenius, S. (2004): Mitochondrial regulation of apoptotic cell death. Toxicol. Lett., 149, 19-23.

Pognan, F. (2004): Genomics, proteomics and metabonomics in toxicology: hopefully not "fashionomics". 5, 879-893.

Raghow, R. (1994): The role of extracellular matrix in postinflammatory wound healing and fibrosis. FASEB J., 8, 823-831.

Rhee, S.G., Kang, S.W., Jeong, W. Chang, T.S., Yang, K.S. and Woo, H.A. (2005): Intracellular messenger function of hydrogen peroxide and its regulation by peroxiredoxins. Curr. Opin. Cell Biol. 17, 183-189.

Roth, R.A., Luyendyk, J.P., Maddox, J.F. and Ganey P.E. (2003): Inflammation and drug idiosyncrasy--is there a connection? J. Pharmacol. Exp. Ther., 307, 1-8.

Sugiyama, Y., Ito, K., Tsuda, M. and Horii, I. (1996): Future prospects for toxicokinetics: its ability to predict drug adverse events in humans. J. Toxicol. Sci., 21, 511-516.

Toivola, D.M. and Eriksson, J.E. (1999): Toxins affecting cell signalling and alteration of cytoskeletal structure. Toxicol. In Vit- 
Darwinian toxicology thought in safety evaluation of drug

ro, 13, 521-530.

Wallace, K.B. and Starkov, A.A. (2000): Mitochondrial targets of drug toxicity. Annu. Rev. Pharmacol. Toxicol., 40, 353-388.

Waring, J.F. and Ulrich R.G. (2000): The impact of genomics-based technologies on drug safety evaluation. Ann. Rev. Pharmacol. Toxicol., 40, 335-352.

Waters, M. and Yauk, C. (2007): Consensus recommendations to promote and advance predictive systems toxicology and toxicogenomics. Environ. Mol. Mutagen., 48, 400-403.
Waters, M.D. and Fostel, J.M. (2004): Toxicogenomics and systems toxicology: aims and prospects. Nat. Rev. Genet., 5, 936-948.

Williams, G.M. (2001): Mechanisms of chemical carcinogenesis and application to human cancer risk assessment. Toxicology, 166, 3-10.

Wyllie, A.H. (1997): Apoptosis: An overview. Br. Med. Bull., 53, 451-465.

Zimmer, C. (2007): Evolution Jurassic genome. Science, 315, 13581359. 
